Bio - grafía. Escritos sobre la Biología y su Enseñanza. ISSN 2027-1034

Edición Extraordinaria. p.p. $610-618$

Memorias del IX Encuentro Nacional de Experiencias en Enseñanza de la Biología y la Educación Ambiental. IV Congreso Nacional de Investigación en Enseñanza de la Biología.

\title{
FORMACION DE JOVENES COMO LÍDERES AMBIENTALES: EL CASO DEL C.E.D LA CONCEPCION DE BOGOTA
}

\author{
IMPORTANCE OF TRAINING YOUNG PEOPLE AS ENVIRONMENTAL \\ LEADERS: THE CASE OF C.E.D THE CONCEPTION OF BOGOTA
}

\author{
Oscar Hernando García Cadena ${ }^{1}$ \\ Jenny Johanna Duarte Díaz ${ }^{2}$
}

\section{RESUMEN}

Conducir la educación ambiental hacia un ejercicio meramente academicista en el aula, o por actores que no están inmersos en las realidades educativas, ha hecho que la aplicabilidad de los conocimientos y las mismas reflexiones se distancien de la cotidianidad de los estudiantes. En este escrito pretendemos mostrar la importancia de la formación de líderes ambientales en la escuela profundizando en el desarrollo de una experiencia, en una institución educativa distrital. La metodología cualitativa se desarrolla desde el enfoque de investigación acción con sujetos de la comunidad educativa inmersos en los procesos de aprendizaje. Determinando como resultado que los estudiantes lideres ambientales como sujetos inmersos en los procesos de aprendizaje, durante el desarrollo y socialización de herramientas comunicativas de educación ambiental, garantizan en los diferentes miembros de la comunidad, el reconocimiento de sus problemáticas ambientales logrando un cambio de perspectiva frente a la educación ambiental. Lo que resultado aplicable a cualquier tipo de población ya que en los diferentes contextos se identifican problemáticas ambientales similares en las que la escuela puede pensar en realizar una intervención que varíe las formas tradicionales de trabajo en educación ambiental.

PALABRAS CLAVE Formación líderes, educación ambiental en la escuela

\section{ABSTRACT}

Conducting environmental education towards a merely academic exercise in the classroom, or for actors who are not immersed in educational realities, has made the applicability of knowledge and the same reflections distanced themselves from the everyday life of students. In this paper, we intend to show the importance of the

\footnotetext{
${ }^{1}$ Licenciado en Educación Física. Estudiante de Maestría de Ciencias y Tecnologías del deporte y actividad Física. Universidad Manuela Beltrán. Docente Educación Física y Deportes. Secretaria de educación Distrital. CED La Concepción. oscargarciacadena@hotmail.com

${ }^{2}$ Estudiante Doctorado interinstitucional en educación. Docente Secretaría de Educación Distrital.
} 
Bio - grafía. Escritos sobre la Biología y su Enseñanza. ISSN 2027-1034

Edición Extraordinaria. p.p. $610-618$

Memorias del IX Encuentro Nacional de Experiencias en Enseñanza de la Biología y la Educación Ambiental. IV Congreso Nacional de Investigación en Enseñanza de la Biología.

formation of environmental leaders in the school, deepening the deve lopment of an experience, in a district educational institution. The qualitative methodology is developed from the action research approach with subjects of the educational community immersed in the learning processes. Determining as a result that the student's environmental leaders in the development and socialization of communicative tools, guarantee in the different members of the community, the recognition of their environmental problems achieving a change of perspective against environmental education. The result that can be applied to any type of population since different environmental problems are identified in the different contexts in which the school can think of an intervention that varies traditional forms of work in environmental education.

KEY WORDS Training leaders, environmental education at school

\section{INTRODUCCION}

La educación ambiental en la escuela debe establecerse desde propuestas diferentes a las tradicionales que partan de diversos campos del saber y no se centren en la realización de actividades desligadas de un propósito de enseñanza. Propuestas en la que se les dé cabida a los jóvenes estudiantes, participes activos de la educación, reconociéndolos como agentes que inciden en el presente y no dando continuidad a la idea que son solo el futuro. Esto indiscutiblemente dará mayor relevancia a su formación como actores políticos y agentes implícitos en una sociedad cumpliendo un rol significativo, identificando que a la juventud históricamente se ha configurado en diversos escenarios, logrando establecer una identidad social que se construye con el apoyo de instituciones de formación como la escuela, la familia o la iglesia y que esta configuración del joven como sujeto social lo lleva a la forma de instaurar sus propias capacidades.

De esta forma, en una sociedad permeada por problemáticas sociales en las que los jóvenes están inmersos, es necesario generarles espacios que les permita identificar esas problemáticas desde una visión crítica y los motive a diseñar estrategias para ser parte de la solución. Así, lograr establecer en ellos como seres humanos el reconocimiento desde la mirada del otro lo que genera la necesidad de establecerse como un ejemplo social, para nuestro caso en términos ambientales.

En esta idea, la formación de liderazgo en la juventud se convierte en un aspecto de suma importancia, al ser un elemento que podría permitir a las sociedades la transformación de sus realidades y la generación de un mayor sentido de pertenencia, consiguiendo al mismo tiempo la re significación de las realidades en los diversos contextos. Sin embargo, se debe aclarar que no podemos hablar de la 
Bio - grafía. Escritos sobre la Biología y su Enseñanza. ISSN 2027-1034

Edición Extraordinaria. p.p. $610-618$

Memorias del IX Encuentro Nacional de Experiencias en Enseñanza de la Biología y la Educación Ambiental. IV Congreso Nacional de Investigación en Enseñanza de la Biología.

juventud en singular, puesto que esto supone considerarla como un sujeto homogéneo que reconoce una sola forma de ser del joven, si no que debemos hablar de juventud en plural (Bonvillani et al. 2010) ya que los jóvenes son muy diferentes entre sí, aunque compartan escenarios y tensiones que les configuran necesidades que son potenciales puntos de encuentro. De esta forma la construcción de liderazgo en la juventud, sin desconocer sus diferencias como individuos pueda verse como un proceso importante en la transformación de realidades de las sociedades actuales.

En este texto, pretendemos mostrar el caso del Centro Educativo Distrital La Concepción, ubicado en la localidad e Bosa, en la ciudad de Bogotá, Colombia. En donde, la propuesta de formación de líderes ambientales en la escuela surgió como una alternativa para retomar este campo desde una perspectiva diferente a la tradicional, buscando que sean los propios estudiantes como líderes empoderados de un saber ambiental, quienes construyan e implementen herramientas para su enseñanza en la escuela. Esta propuesta se construye reconociendo que cuando el mensaje es trasmitido por pares, resulta ser mucho más significativo.

Bajo esta idea, se ha identificado que la propuesta ha permitido establecer desde la reflexión un sentido de pertenencia y apropiación del ambiente y a la vez ha generado una mayor incidencia en cuanto al cambio de actitudes, en los diversos miembros, en relación a aquellos ejercicios de educación ambiental que tradicionalmente se venían adelantando desde el aula, los cuales particularmente estaban limitados a la disciplina de las Ciencias Naturales.

La experiencia que se describirá, se vincula a las políticas distritales que proponen "promover acciones pedagógicas que permitan propiciar proyectos educativos que incidan, no solo en el nivel institucional, sino también en la transformación de las realidades ambientales de su entorno" (SED, 2013). Además, se ha tenido en cuenta el Proyecto Educativo Institucional del colegio, que propende por "ofrecer una formación integral que favorezca el desarrollo de una gestión en que se den las condiciones necesarias para el ejercicio progresivo de competencias que potencien la formación de ciudadanos líderes capaces de razonar, debatir, producir y responder con acierto a la problemática personal, familiar, ambiental y social de la comunidad" (Documento PEI, 2009).

El objetivo esencial de la propuesta ha sido variar las formas tradicionales de abordar la educación ambiental en la escuela, generando el empoderamiento de jóvenes como líderes ambientales bajo la idea de la educación para la ciudadanía, la cual expone que se debe promover el empoderamiento de los y las jóvenes, que comprenda el desarrollo de habilidades cognitivas y reflexivas (Sánchez, 2012). 
Bio - grafía. Escritos sobre la Biología y su Enseñanza. ISSN 2027-1034

Edición Extraordinaria. p.p. $610-618$

Memorias del IX Encuentro Nacional de Experiencias en Enseñanza de la Biología y la

Educación Ambiental. IV Congreso Nacional de Investigación en Enseñanza de la Biología.

\section{METODOLOGIA}

En relación al desarrollo metodológico, se generó desde un enfoque interpretativo, una propuesta escolar apoyada en la idea de aprendizaje colaborativo, entendido como un proceso de aprendizaje que enfatiza en los esfuerzos colaborativos entre profesores y estudiantes, a su vez, que es facilitado por la interacción social en un entorno que permite la cooperación y evaluación entre iguales (Hilz y Turoff, 1993).

Además, la propuesta se direcciono desde la idea de la Investigación Acción, bajo la postura de Kemmis (Paramo, 2011) quien la explica como una forma de indagación autorreflexiva realizada por quienes participan en las situaciones sociales para mejorar la racionalidad de a) sus propias prácticas sociales 0 educativas; b) su comprensión sobre las mismas; y c) las situaciones e instituciones en que estas prácticas se realizan (aulas o escuelas). Asumimos este tipo de investigación por ser un proceso de carácter cíclico dialéctico, como consecuencia de la relación entre la acción y la reflexión, lo que corresponde totalmente con nuestra idea.

En el desarrollo de esta propuesta los principales recursos humanos han estado dados en los jóvenes líderes ambientales integrantes del comité ambiental y estudiantes del colegio La Concepción ubicado en la localidad de Bosa, estos han sido multiplicadores del mensaje ambiental con sus compañeros, los cuales a su vez han sido replicadores con los miembros de su familia, vecinos y las personas que habitan cerca de la institución, generando así un gran impacto social y permitiendo que los estudiantes integrantes de la propuesta se conviertan en sujetos críticos que proponen soluciones a las problemáticas de su comunidad. Se debe mencionar que la experiencia se ha adelantado en espacios totalmente diferentes a las aulas de clase en donde los tiempos de reuniones y actividades con estudiantes se han acordado en extra jornada, esto a fin de no centrar la educación ambiental a contenidos propios de algunos conocimientos disciplinares.

Para el desarrollo de la propuesta se han generado diversas etapas, algunas de las cuales se han dado secuencialmente y otras de forma simultánea. La primera etapa se describió en identificar como se abordaba la educación ambiental en la escuela.

Posteriormente fue necesario en primera instancia realizar un proceso de formación con los estudiantes que mostraban intereses de pertenecer al grupo de líderes ambientales en la institución. Seguidamente, surgió la idea de construir una herramienta innovadora desde lo audiovisual y visual como un súper héroe ambiental, que tuvo como objetivo inicial presentarse a la comunidad educativa e invitar a los estudiantes a ser parte del grupo ambiental institucional. 
Bio - grafía. Escritos sobre la Biología y su Enseñanza. ISSN 2027-1034

Edición Extraordinaria. p.p. $610-618$

Memorias del IX Encuentro Nacional de Experiencias en Enseñanza de la Biología y la

Educación Ambiental. IV Congreso Nacional de Investigación en Enseñanza de la Biología.

Con los jóvenes participantes, se construyó una miniserie y una cartilla relacionada al super héroe ambiental, para la generación de conciencia en la institución educativa, los líderes ambientales iniciaron una nueva fase, establecida en el proceso de socialización de la mini serie con sus compañeros. Para esto se organizaron jornadas institucionales ambientales en las que se realizaron proyecciones en las aulas de sus diferentes capítulos. Adicional a mostrar la miniserie, en estos espacios los lideres ambientales aprovechaban para contar sus experiencias personales frente al proceso de formación ambiental y construcción de la producción, lo que generó mucho interés en la comunidad vinculando a otros estudiantes, padres de familia y docentes. El diseño de las herramientas estuvo a cargo de los jóvenes líderes ambientales, buscando que con el mensaje que ellos pusieran a estas herramientas los niños se concientizaran de las problemáticas ambientales y generaran el cuidado de los recursos. Estas herramientas fueron abordadas por los líderes ambientales inicialmente con los niños pertenecientes a la educación básica primaria buscando desde sus motivaciones despertar en los estudiantes de cortas edades que observen a sus compañeros como un ejemplo a seguir reconociendo en ellos diferentes capacidades.

Como actividad significativa que vincula a otros miembros de la comunidad, profesores invitan a los líderes ambientales a trabajar en sus clases haciendo parte activa de las mismas, con espacios donde pueden exponer los trabajos realizados con otros grupos, algunos de los profesores que han vinculado a los líderes ambientales a su clases son los de las áreas de sociales, preescolar y ciencias naturales, ellos han incluido dentro de sus discursos cotidianos el trabajo ambiental desde sus aulas de clase utilizando como herramienta didáctica la socialización por parte de los lideres ambientales de la cartilla, como ejemplos podemos mencionar un profesor de sociales que presenta una relación de la herramienta del súper héroe ambiental con la transformación de las diversas culturas y su incidencia en el ambiente, él se proponía mostrar el cambio que ha tenido el ser humano en su relación con el ambiente hasta la actualidad apoyado en el discurso de los lideres ambientales, algunas maestras de preescolar han utilizado el proceso de los líderes ambientales para trabajar desde las diferentes dimensiones el reconocimiento de los recursos naturales con el recurso de la cartilla. En estos ejercicios formativos vinculados a contenidos particulares los jóvenes líderes ambientales han sido más que acompañantes, los que direccionan la educación ambiental, con acciones como la persuasión frente al cuidado de los recursos que utilizan todos los días.

Las herramientas, se diseñaron a partir de la recolección de las ideas de los estudiantes que los mismos líderes recogieron. Lo que se realizó con una 
Bio - grafía. Escritos sobre la Biología y su Enseñanza. ISSN 2027-1034

Edición Extraordinaria. p.p. $610-618$

Memorias del IX Encuentro Nacional de Experiencias en Enseñanza de la Biología y la Educación Ambiental. IV Congreso Nacional de Investigación en Enseñanza de la Biología.

encuesta, en la que los líderes ambientales indagaron a los estudiantes frente a los intereses para trabajar la educación ambiental en la escuela y el como propiciar la continuidad de la historieta del capitán ambiente, consolidando la información para el diseño de las cartillas y su implementación. Es necesario mencionar que para el desarrollo de la propuesta la institución educativa ha sido un gran apoyo porque con su financiamiento en la mayor parte de actividades y el suministro de elementos tecnológicos como cámaras de video, fotográficas, computadores, prendas de vestir alusivas al proyecto, entre otros, ha facilitado el desarrollo de las acciones propuestas permitiendo el reconocimiento de los estudiantes ante la comunidad educativa.

\section{RESULTADOS}

Frente a los efectos obtenidos con el desarrollo de la propuesta de educación ambiental debemos iniciar mencionado que en primer lugar obtuvimos la identificación de la forma en la que la institución educativa había venido abordando la educación ambiental, a fin de establecer y delimitar la idea. Para esto, nos apoyamos en el planteamiento de González (2000) quien expone un momento de la dimensión ambiental en el contexto escolar referido a la ambientalización de las ciencias naturales, en el que se propone añadir contenidos relacionados con el ambiente a esta área particular. En este momento, encontramos estaba enmarcada la educación ambiental en el Colegio La Concepción, ya que su trabajo se había limitado a utilizar el reconocimiento de espacios naturales y sus interacciones biológicas físicas y químicas. Se pudo evidenciar que la comunidad educativa estaba permeada por la educación ambiental que se abordaba de forma tradicional desde las clases de ciencias naturales, por lo que, se identificó que esta podría ser más asertiva en sus fines, cuando eran los mismos estudiantes los que llevaran el mensaje y más motivante al implicar medios de comunicación contextuales para su enseñanza. De esta manera se replanteo la forma en la que se trabajaría el mensaje ambiental en la escuela.

De esta forma, al plantear propuestas diferentes se generó la intervención activa de muchos estudiantes que deseaban ser líderes ambientales, es decir, los jóvenes se sintieron identificados aumentando el sentido de pertenencia en relación a su entorno, la motivación por ser líderes ambientales, y por ende la multiplicación del mensaje en la comunidad. Además, como resultado vinculado directamente al objetivo de la propuesta de líderes ambientales que realizan procesos de formación, se percibió en la comunidad educativa un gran impacto identificando que estudiantes, profesores y padres de familia reconocen las nuevas formas de educación ambiental en su escuela; motivándose a continuar 
Bio - grafía. Escritos sobre la Biología y su Enseñanza. ISSN 2027-1034

Edición Extraordinaria. p.p. $610-618$

Memorias del IX Encuentro Nacional de Experiencias en Enseñanza de la Biología y la Educación Ambiental. IV Congreso Nacional de Investigación en Enseñanza de la Biología.

con el trabajo de recuperación y apropiación del entorno e identificando que ahora sus problemáticas ambientales pueden ser solucionables con acciones colectivas.

Dentro del proceso de evaluación y seguimiento de la propuesta el trabajo realizado se abordó bajo la secuencia cíclica (Figura 1) que se contempla desde la idea de Investigación Acción la cual ha llevado a la transformación social de la escuela frente a la percepción de ambiente incluyendo al ser humano como parte de este.

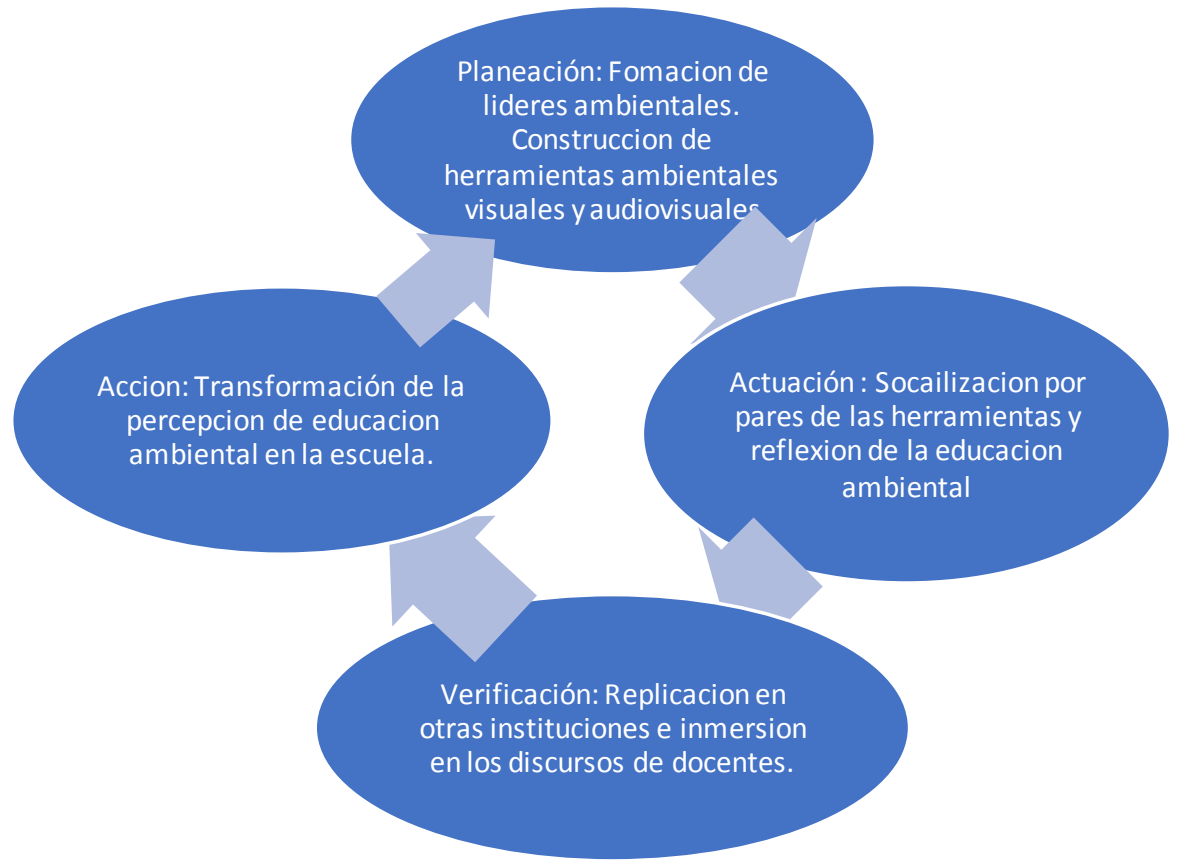

Figura 1. Evaluación y seguimiento de la propuesta

En el desarrollo de la experiencia existió siempre un proceso de auto evaluación de cada una de las acciones realizadas lo cual permitió evidenciar y ser conscientes de aquello que se ha hecho de manera adecuada y de lo que se debe replantear. De esta manera se ha valorado si el mensaje que llevan los lideres ambientales permea a los diferentes miembros de la comunidad o si las acciones se presentaban de forma tradicional y repetitivas, generando el surgimiento de nuevas ideas, para llegar a ellos de una forma más interesante y motivadora.

\section{DISCUSION}

La revisión de aspectos en los que se posicionaba la educación ambiental en este tipo de comunidad educativa nos permitió concluir que la formulación del mensaje 
Bio - grafía. Escritos sobre la Biología y su Enseñanza. ISSN 2027-1034

Edición Extraordinaria. p.p. $610-618$

Memorias del IX Encuentro Nacional de Experiencias en Enseñanza de la Biología y la Educación Ambiental. IV Congreso Nacional de Investigación en Enseñanza de la Biología.

que se quiere enseñar en términos de educación ambiental en muchas ocasiones no tiene una conexión desde las políticas y la forma de abordarla, por lo que resulta siendo poco apropiada. Así, se hace necesario variar las formas tradicionales de educación ambiental en la escuela, estableciéndolas de forma independientes al área de conocimiento o formación de los maestros que cotidianamente asumen este reto. Teniendo claro, que no podemos limitarnos a trabajar en las problemáticas ambientales que nos aquejan desde la misma óptica.

Finalmente, se identifica que la propuesta de formación líderes ambientales ha generado un gran impacto en la comunidad educativa resaltando que los jóvenes se presentan como sujetos propositivos generadores de conciencia ambiental y multiplicadores de ideales de conservación de los recursos, propiciando motivación de los estudiantes para recuperar su entorno; concientización de las familias frente a las problemáticas ambientales de su entorno; además desarrollan el deseo de nuevos estudiantes para pertenecer al proyecto ambiental.

\section{CONCLUSIÓN}

Dentro de la formación de personas críticas y empoderadas de las causas ambientales es importante trabajar en su formación como líderes desde la escuela, utilizando nuevas posibilidades de enseñanza que les permitan poner en práctica un discurso actual y realista de las condiciones ambientales de su entorno más cercano y de las problemáticas mundiales.

\section{BIBLIOGRAFIA}

González, E. (2000). La transversalidad de la Educación Ambiental en el curriculum de la enseñanza básica. Reflexiones sobre la educación ambiental II. Carpeta Informativa CENEAM. Edita, organismo autónomo Parques Naturales y Ministerio de Medio Ambiente.

Bonvillani, A., Palermo, A., Vázquez, M., Vommaro, P. (2010). Del Cordobazo al kirchnerismo. Una lectura crítica acerca de los periodos, temáticas y perspectivas de los estudios sobre juventudes y participación política en Argentina. En S. Alvarado y P. Vommaro, Jóvenes, cultura y política en América Latina: algunos trayectos de sus relaciones, experiencias y lectura (1960-2000) (pp. 21-54). Buenos Aires: Clacso.

Hilzt y Turoff, 1993. Tomado de Entornos virtuales fde enseñanza aprendizaje: El proyecto GET. 
Bio - grafía. Escritos sobre la Biología y su Enseñanza. ISSN 2027-1034

Edición Extraordinaria. p.p. $610-618$

Memorias del IX Encuentro Nacional de Experiencias en Enseñanza de la Biología y la Educación Ambiental. IV Congreso Nacional de Investigación en Enseñanza de la Biología.

Secretaria de Educación del Distrito - SED. (2012). Proyecto de Educación para la Ciudadanía y la Convivencia (PECC).

Paramo, P. (2011) La investigación en Ciencias Sociales: estrategias de investigación.

Proyecto Educativo Institucional (2009). La Concepción hacia el futuro. Construyendo hechos de vida. CED. La Concepción. Bogotá. 